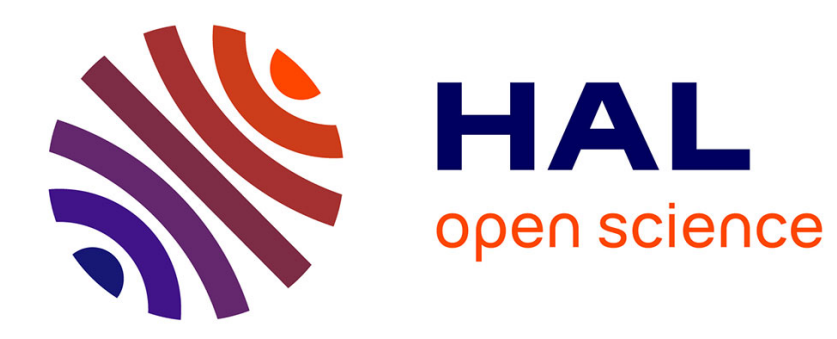

\title{
Non-equilibrium dissociation of nitrogen
}

M. Capitelli, M. Dilonardo

\section{To cite this version:}

M. Capitelli, M. Dilonardo. Non-equilibrium dissociation of nitrogen. Revue de Physique Appliquée, 1978, 13 (3), pp.115-123. 10.1051/rphysap:01978001303011500 . jpa-00244429

\section{HAL Id: jpa-00244429 https://hal.science/jpa-00244429}

Submitted on 1 Jan 1978

HAL is a multi-disciplinary open access archive for the deposit and dissemination of scientific research documents, whether they are published or not. The documents may come from teaching and research institutions in France or abroad, or from public or private research centers.
L'archive ouverte pluridisciplinaire HAL, est destinée au dépôt et à la diffusion de documents scientifiques de niveau recherche, publiés ou non, émanant des établissements d'enseignement et de recherche français ou étrangers, des laboratoires publics ou privés. 
Classification

Physics Abstracts

$52.20 \mathrm{H}-82.20 \mathrm{M}-82.20 \mathrm{R}$

\title{
NON-EQUILIBRIUM DISSOCIATION OF NITROGEN
}

\author{
M. CAPITELLI and M. DILONARDO \\ Centro di Studio per la Chimica dei Plasmi del C. N. R., Dipartimento di Chimica, \\ Università di Bari, Via G. Amendola 173, 70126 Bari, Italy \\ (Reçu le 30 septembre 1977, révisé le 5 décembre 1977 et accepté le 6 décembre 1977)
}

\begin{abstract}
Résumé. - Les vitesses de dissociation de l'azote moléculaire ont été calculées en résolvant un système de master équations comprenant les transferts d'énergie V-V (vibration-vibration), V-T (vibration-translation) et e-V (électron-vibration).

Les résultats montrent que les vitesses de dissociation augmentent légèrement lorsque la température du gas décroît dans l'interval de température $1000-2000 \mathrm{~K}$ pour une température des électrons $T_{\mathrm{e}}=20000 \mathrm{~K}$ et pour une densité des électrons $n_{\mathrm{e}}=10^{12} \mathrm{~cm}^{-3}$. Dans ce calcul nous avons montré que les vitesses de dissociation sont de deux ordres de grandeur plus élevées que celles obtenues par impact direct d'électrons.

Abstract. - Dissociation rates of molecular nitrogen in electrical discharges have been calculated by solving a system of master equations, including V-V (vibration-vibration), V-T (vibrationtranslation) and e-V (electron-vibration) energy transfers.

The results show that the dissociation rates slightly increase with decreasing gas temperature in the temperature range $1000-2000 \mathrm{~K}$ at the electron temperature $T_{\mathrm{e}}=20000 \mathrm{~K}$ and electron density $n_{\mathrm{e}}=10^{12} \mathrm{~cm}^{-3}$. In these calculations, dissociation rates are two orders of magnitude greater than the corresponding rates obtained by direct electron impact.
\end{abstract}

1. Introduction. - We have recently proposed a a theoretical model to explain the high dissociation rates of molecular hydrogen in electrical discharges [1]. The model is based on a solution of the master equation for the populations of the vibrational states of the ground electronic state. Processes considered include vibration-vibration (V-V), vibration-translation (V-T) and electron-vibration (e-V) $\left(^{1}\right)$ energy exchanges. In the case of molecular hydrogen, at low electron temperature $\left(k T_{\mathrm{e}} \simeq \mathrm{leV}\right)$, this model predicts dissociation rates greater than those obtained by direct electronic impact :

$$
\mathrm{e}+\mathrm{H}_{2} \rightleftarrows \mathrm{H}_{2}^{*}+\mathrm{e} \rightleftarrows 2 \mathrm{H}+\mathrm{e}
$$

while at higher electron temperatures the two models predict dissociation rates of the same order of magnitude.

These rates were explained on the basis of an anharmonic pumping of higher vibrational levels by $\mathrm{V}-\mathrm{V}$ and $\mathrm{e}-\mathrm{V}$ energy transfers. The $\mathrm{V}-\mathrm{T}$ processes, on the contrary, tend to depopulate the higher vibrational levels, thereby reducing the dissociation rates. The disso-

(1) The notation (e-V) should not be confused with the corresponding one indicating the transfer of electronic and vibrational energies. ciation rates were found to increase with decreasing gas temperature and pressure, due to the fact that both these parameters tend to increase the V-T energy exchanges. In this work we extend these calculations to pure nitrogen discharges. The choice of $\mathrm{N}_{2}$ was motivated by the fact that the $\mathrm{V}-\mathrm{T}$ rates of $\mathrm{N}_{2}-\mathrm{N}_{2}$ collisions are several orders of magnitude lower than

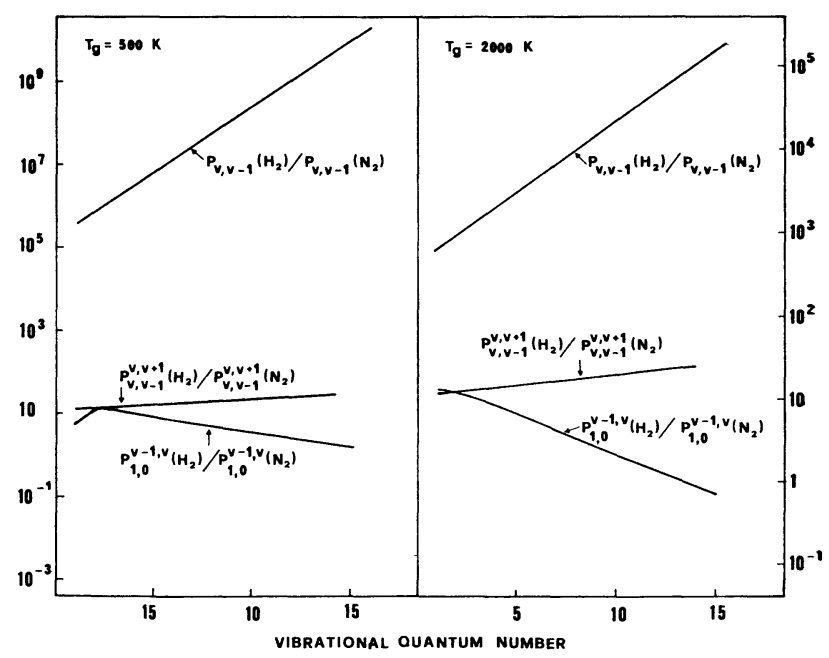

Fig. 1. - A comparison of V-V and V-T rate coefficients (nondimensional) for $\mathrm{H}_{2}$ and $\mathrm{N}_{2}$ systems. 
the values for $\mathrm{H}_{2}-\mathrm{H}_{2}$ at the corresponding vibrational quantum numbers, the other rates (i.e. V-V and e-V) being not too much different (see Fig. 1).

The proposed model, therefore, should predict $\mathrm{N}_{2}$ dissociation rates higher than the corresponding ones for $\mathrm{H}_{2}$, a result completely different from that occurring in the thermal region. The results apply to mode- rate pressure (5-50 torr) r.f. discharges, characterized by $E / N$ values ranging from $10^{-16}$ to $10^{-15} \mathrm{~V} . \mathrm{cm}^{2}$ and electron densities from $10^{10}$ to $10^{12} \mathrm{~cm}^{-3}$.

2. Method of calculation. - The dissociation rate $v_{\mathrm{d}}$ (particles $/ \mathrm{cm}^{3} \mathrm{~s}$ ) of a diatomic species in an electrical discharge can be written as:

$$
\begin{aligned}
v_{\mathrm{d}} & =\partial N_{v^{\prime}+1} / \partial t=-\sum_{0}^{v^{\prime}} \partial N_{v} / \partial t=k_{\mathrm{d}}(t) \sum_{0}^{v^{\prime}} N_{v} \\
& =N_{\mathrm{N}_{2}} N_{v^{\prime}} P_{v^{\prime}, v^{\prime}+1}+n_{\mathrm{e}} \sum_{0}^{v^{\prime}} N_{v} K_{v, v^{\prime}+1}^{\mathrm{e}}+\sum_{1}^{v^{\prime}} N_{v} P_{v, v-1}^{v^{\prime}, v^{\prime}+1} N_{v^{\prime}}
\end{aligned}
$$

where $N_{v^{\prime}+1}$ represents the number density of a pseudolevel above the last vibrational level $\left(v^{\prime}\right)$ of the ground electronic state of $\mathrm{N}_{2}, k_{\mathrm{d}}$ is the dissociation rate constant $\left(\mathrm{s}^{-1}\right), N_{\mathrm{N}_{2}}$ and $n_{\mathrm{e}}$ are the nitrogen and electron number densities.

Equation (2) is coupled to a system of $v^{\prime}$ master equations of the type

$$
\begin{aligned}
\partial N_{v} / \partial t=P_{v+1, v} N_{\mathrm{N}_{2}}\{ & \left.N_{v+1}-\exp \left[-\left(E_{v+1}-E_{v}\right) / k T_{\mathrm{g}}\right] N_{v}\right\} \\
& -P_{v, v-1} N_{\mathrm{N}_{2}}\left\{N_{v}-\exp \left[-\left(E_{v}-E_{v-1}\right) / k T_{\mathrm{g}}\right] N_{v+1}\right\} \\
& +\sum_{w=1}^{v^{\prime}+1} P_{v+1, v}^{w-1, w}\left\{N_{v+1} N_{w-1}-\exp \left[-\left(E_{v+1}+E_{w-1}-E_{v}-E_{w}\right) / k T_{\mathrm{g}}\right] N_{v} N_{w}\right\} \\
& -\sum_{w=0}^{v^{\prime}} P_{v, v-1}^{w, w+1}\left\{N_{v} N_{w}-\exp \left[-\left(E_{v}+E_{w}-E_{w+1}-E_{v-1}\right) / k T_{\mathrm{g}}\right] N_{v-1} N_{w+1}\right\} \\
& +n_{\mathrm{e}} \sum_{w=0}^{v^{\prime}} K_{w v}^{\mathrm{e}}\left\{N_{w}-\exp \left[-\left(E_{w}-E_{v}\right) / k T_{\mathrm{e}}\right] N_{v}\right\}
\end{aligned}
$$

where $P_{v+1, v}, P_{v, v-1}, P_{v+1, v}^{w-1, w} P_{v, v-1}^{w, w+1}$ and $K_{w v}^{\mathrm{e}}$ represent the rate coefficients of the following microscopic processes

$$
\begin{aligned}
& \left.\begin{array}{l}
N_{2}+N_{2}(v+1) \rightleftarrows N_{2}+N_{2}(v) \\
N_{2}+N_{2}(v) \rightleftarrows N_{2}+N_{2}(v-1)
\end{array}\right] \mathrm{V}-\mathrm{T} \\
& \left.\begin{array}{l}
N_{2}(v+1)+N_{2}(w-1) \rightleftarrows N_{2}(v)+N_{2}(w) \\
N_{2}(v)+N_{2}(w) \rightleftarrows N_{2}(v-1)+N_{2}(w+1)
\end{array}\right] \mathrm{V}-\mathrm{V} \\
& \left.N_{2}(w)+\mathrm{e} \rightleftarrows N_{2}(v)+\mathrm{e} \quad\right] \mathrm{e}-\mathrm{V}
\end{aligned}
$$

$T_{\mathrm{e}}$ and $T_{\mathrm{g}}$ represent the electron and gas temperatures respectively.

It should be noted that the present mechanism does not consider the electron impact dissociation, the importance of which will be considered in the next section.

The system of $46+1$ differential equations can be solved once one knows : $a$ ) the vibrational energies ; b) the V-T, V-V and e-V rate constants.

An anharmonic Morse oscillator $\left(E_{v}=E_{10}\left[\left(v+\frac{1}{2}\right)-\delta\left(v+\frac{1}{2}\right)^{2}\right]\right)$ has been used for calculating $E_{v}$ $(\delta=$ the anharmonicity constant $=0.006217$ ). The number of vibrational levels including the pseudolevel $\left(v^{\prime}+1\right)$ has been determined as the largest value of $v$ for which $E_{v}$ is less than or equal to the dissociation energy $D$. This value $\left(v^{\prime}+1=46\right)$, which is slightly different from the corresponding one reported by Kewley [2] $\left(v^{\prime}+1=44\right)$ or by Bray [3] $\left(v^{\prime}+1=47\right)$ should not affect the present calculations as pointed out in section $4\left({ }^{2}\right)$.

(2) It should be noted that $v^{\prime}$ calculated in the present work strongly differs from that calculated by using the Herzberg formula : $v^{\prime}=1 / 2 \delta \simeq 80$. This last value gives a dissociation energy 1.23 times greater than the experimental value. It should be noted that the system of $46+1$ equations refers to 46 vibrational levels $(v=0, \ldots, v=45)$ plus the pseudolevel. 
The modified SSH theory $[3,4]$ has been used for calculating the V-T and V-V rate coefficients $\left(\mathrm{cm}^{3} / \mathrm{s}\right)$, which appear in the form

$P_{v, v-1}=Z_{11} P_{11} T_{g}[v /(1-\delta v)] F\left(x_{v, v-1}\right)$

$P_{v, v-1}^{w-1, w}=Z_{11} Q_{11} T_{g}[v /(1-\delta v)][w /(1-\delta w)] F\left(y_{v, v-1}^{w-1, w}\right)$ for $w \leqslant v$

$P_{v, v-1}^{w-1, w}=Z_{11} Q_{11} T_{\mathrm{g}}[v /(1-\delta v)][w /(1-\delta w)] F\left(y_{v, v-1}^{w-1, w}\right) \exp \left[\left(E_{v}+E_{w-1}-E_{v-1}-E_{w}\right) / k T_{\mathrm{g}}\right]$

$x_{v, v-1}=\left(\frac{1}{2}\right)^{3 / 2}\left(\theta_{11}^{\prime} / T_{\mathrm{g}}\right)^{1 / 2}(1-2 \delta v)$

$y_{v, v-1}^{w-1, w}=2 \delta\left(\frac{1}{2}\right)^{3 / 2}\left(\theta_{11}^{\prime} / T_{\mathrm{g}}\right)^{1 / 2}|v-w|$

$\theta_{11}^{\prime}=5.39 \times 10^{6} \mathrm{~K}$

where $Z_{11}$ is the $\mathrm{N}_{2}-\mathrm{N}_{2}$ collision frequency calculated on the basis of a collision diameter $\sigma=3.75 \AA$ (see ref. [2]).

The $P_{11}$ values have been obtained by matching the equations to the experimental correlation data [5].

$(p \tau)_{\mathrm{V}-\mathrm{T}}\left(\mathrm{N}_{2}-\mathrm{N}_{2}\right)=$

$$
=5.7 \times 10^{-12} \exp \left(234.9 T_{\mathrm{g}}^{-1 / 3}\right) \mathrm{atm} . \mathrm{s}
$$

while the $Q_{11}$ ones have been obtained by calculating $P_{0,1}^{1,0}$ by means of equation (22) of reference [6].

Figure 2 reports some of the calculated V-T and $\mathrm{V}-\mathrm{V}$ rate coefficients.

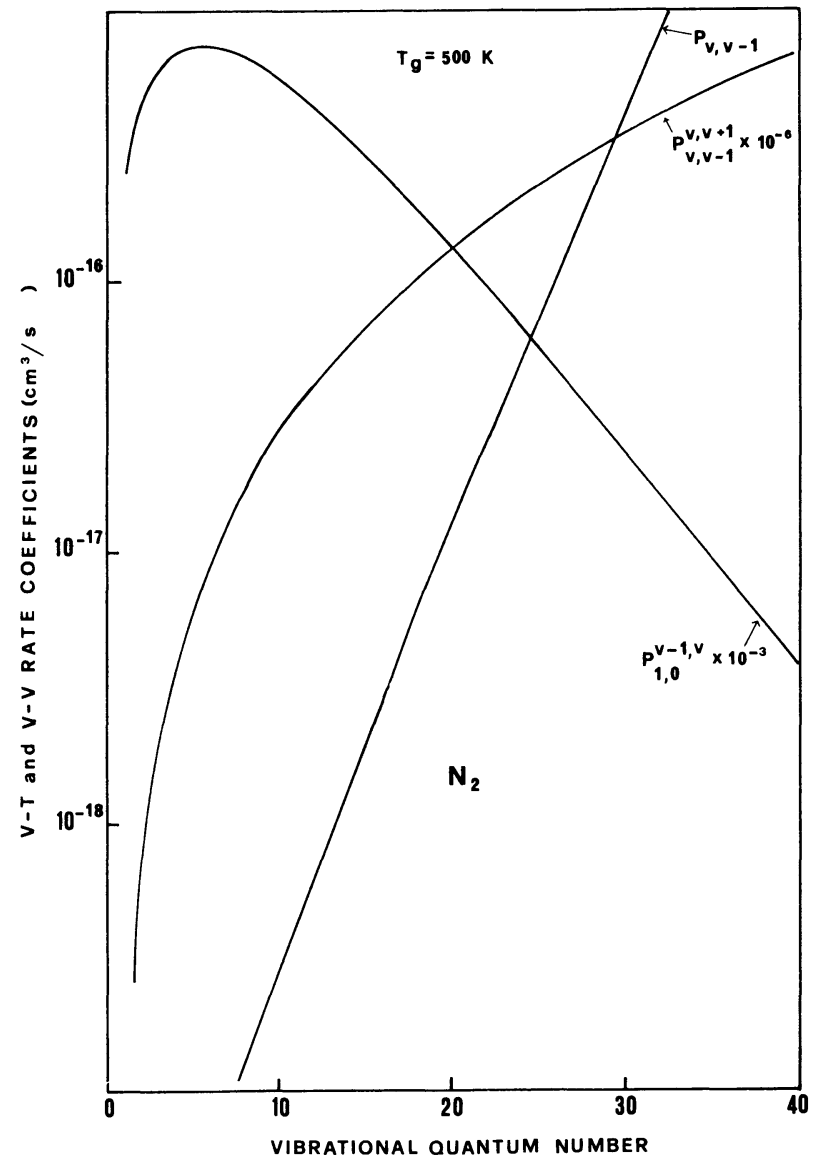

FIG. 2. - Rate coefficients for V-T and V-V processes $\left(\mathrm{cm}^{3} / \mathrm{s}\right)$ as a function of vibrational quantum number for $\mathrm{N}_{2}$.
The e-V rate coefficients have been obtained by numerical integration of the experimental O-v $(v \leqslant 8)$ cross sections [7] over a Maxwellian distribution function for the electrons, while those for $h-v$ transitions $(h>0)$ have been assumed equal to the corresponding O- $v$ transitions (i.e. $K_{0,1}^{\mathrm{e}}=K_{1,2}^{\mathrm{e}}=K_{2,3}^{\mathrm{e}}$ and so on). Values of $K_{0,1}^{\mathrm{e}}$ and of

$$
\left(\sum_{i=1}^{8} K_{0, i}^{\mathrm{e}}\right)
$$

have been reported in table I. The system of differential equations (2-3) written in vector form as

$$
\delta \mathbf{n} / \delta t=\mathbf{f}(\mathbf{n})
$$

has been integrated with the following numerical algorithm [8]

$$
\mathbf{n}(t+h)=\mathbf{n}(t)+\left(\mathbf{I}-\frac{1}{2} h \mathbf{J}+\frac{1}{12} h^{2} \mathbf{J}^{2}\right)^{-1} h \mathbf{f}(\mathbf{n}(t))
$$

where $\mathbf{I}$ is the identity matrix, $\mathbf{J}$ is the jacobian of the system calculated at time $t$ and $h$ is the step size.

TABLE I

Rate coefficients $\left(\mathrm{cm}^{3} / \mathrm{s}\right)$ for e-V processes

$$
\begin{array}{ccccc}
T_{\mathrm{e}}(\mathrm{K}) & K_{0,1}^{\mathrm{e}} & \sum_{i=1}^{8} K_{0, v}^{\mathrm{e}} & K_{\text {eff }}^{\mathrm{e}}(a) & K_{\text {eft }}^{\mathrm{e}}(b) \\
- & - & - & - & - \\
10000 & 1.7(-9) * & 6.1(-9) & 1.3(-8) & 1.1(-8) \\
20000 & 2.4(-9) & 8.8(-9) & 2.6(-8) & 2.2(-8)
\end{array}
$$

$* 1.7(-9)=1.7 \times 10^{-9}$.

$\left({ }^{a}\right)$ Present results.

(b) Reference [16].

Most of the results have been obtained by imposing at $t=0$ the following initial condition :

$$
\begin{aligned}
& N_{v}(t=0)=0 \quad \text { for } v>0 \\
& N_{0}(t=0)=N_{\mathrm{N}_{2}}
\end{aligned}
$$


3. Results. - After a transient period, which is approximately given by $\left(n_{\mathrm{e}} K_{0,1}^{\mathrm{e}}\right)^{-1}, k_{\mathrm{d}}(t)$ settles to a constant value $k_{\mathrm{d}}^{\mathbf{s}}$, the so called steady state dissociation constant (see Fig. 3). A true steady state disso-

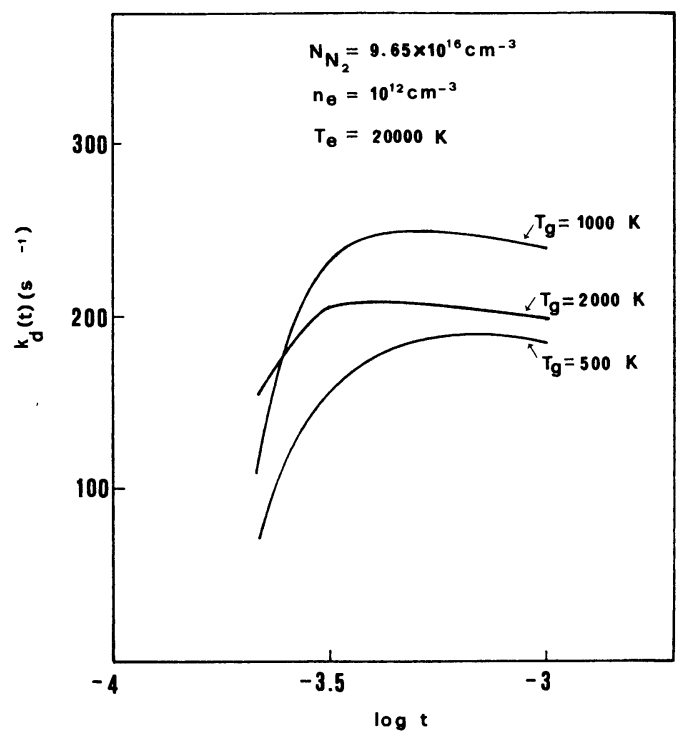

FIG. 3. - Temporal evolution of $k_{\mathrm{d}}(t)$.

ciation constant cannot be achieved in our model because one neglects the recombination process and this yields $k_{\mathrm{d}}(t=\infty)=0$. The $k_{\mathrm{d}}^{\mathrm{s}}$ values reported in the present work are therefore those corresponding to plateaus or maxima of $k_{\mathrm{d}}(t)$ plots $\left(^{3}\right)$.

Values of $\log k_{\mathrm{d}}^{\mathrm{s}}$ have been reported in figure 4 as a function of $1 / T_{\mathrm{g}}$ for $n_{\mathrm{e}}=10^{12} \mathrm{~cm}^{-3}$ and $T_{\mathrm{e}}=20000 \mathrm{~K}$. In the same figure we have also reported the values for $n_{\mathrm{e}}=0$.

It should be noted that the behaviour of $k_{\mathrm{d}}^{\mathrm{s}}$ in the thermal conditions $\left(n_{\mathrm{e}}=0\right)$ is completely different from that occurring in the discharges $\left(n_{\mathrm{e}} \neq 0\right)$.

For thermal conditions the dissociation constant strongly decreases with decreasing gas temperature. An activation energy of $250 \mathrm{kcal} / \mathrm{mole}$ is obtained in this case, which is $10 \%$ higher than the nitrogen dissociation energy. A satisfactory agreement is found between theoretical and experimental $k_{\mathrm{d}}^{\mathrm{s}}$ values $[9,10]$, as can be appreciated from table II. It should be noted that our results are always lower than the experimental ones. An increase of the theoretical dissociation rate is however expected when one considers the rotational and electronic degrees of freedom of diatomic molecule. This inclusion, for example, enhances the theoretical dissociation constant of oxygen in thermal conditions by a factor 9 at $T_{\mathrm{g}}=4500 \mathrm{~K}[11]$.

(3) The $k_{\mathrm{d}}^{\mathbf{s}}$ values reported in this work correspond to a pseudofirst order kinetic equation (see Eq. (2)), even though, due to the prevalence of V-V processes, a second order coefficient would be more revealing. We prefer, however, to keep the definition of equation 2 by analogy with the $\mathrm{H}_{2}$ system of ref. [1].

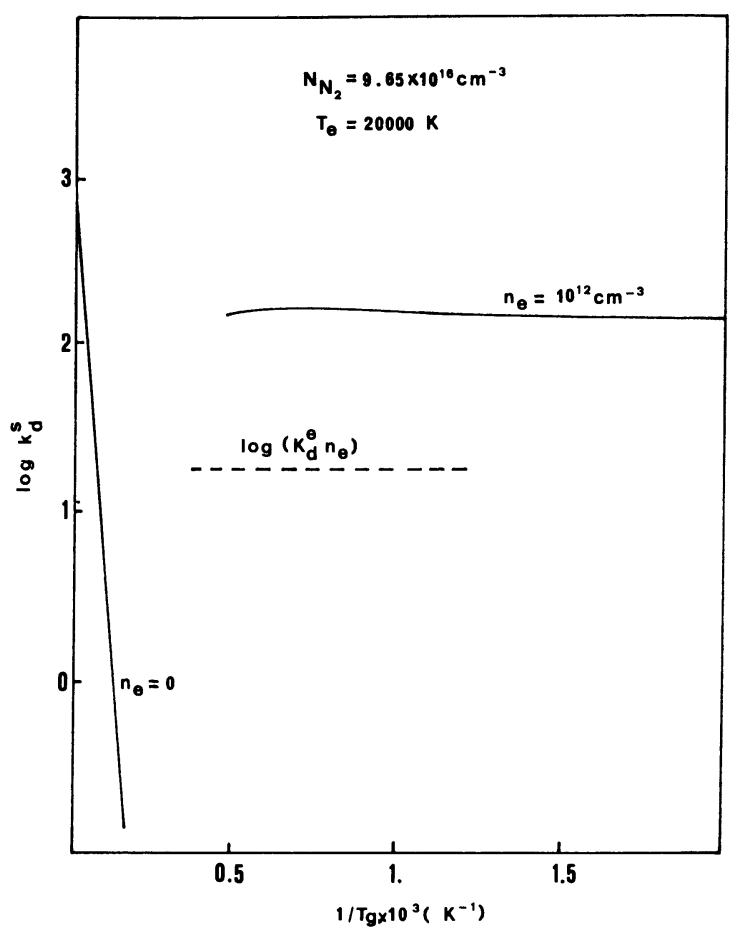

Fig. 4. - Values of $\log k_{\mathrm{d}}^{\mathrm{s}}$ and of $\log \left(k_{\mathrm{d}}^{\mathrm{e}} n_{\mathrm{e}}\right)$ as a function of $1 / T_{\mathrm{g}}$.

\section{TABLE II}

A comparison between theoretical and experimental values of $k_{\mathrm{d}}^{\mathrm{s}}(c c /$ mole.s) for thermal conditions

$$
\left(n_{\mathrm{e}}=0\right)
$$

$$
\begin{aligned}
& T_{\mathrm{g}}(\mathrm{K}) \\
& k_{\mathrm{d}}^{\mathrm{s}}\left({ }^{a}\right) \\
& 6000 \\
& 1.0(+6) \text { * } \\
& k_{\mathrm{d}}^{\mathrm{s}}\left(^{b}\right) \\
& 10000 \\
& 4.5(+9) \\
& 8.8(+7) \\
& 2.8(+10) \\
& k_{\mathrm{d}}^{\mathrm{s}}\left({ }^{c}\right) \\
& 1.8(+10)
\end{aligned}
$$

In the electrical conditions $\left(n_{\mathrm{e}}=10^{12} \mathrm{~cm}^{-3}\right)$ the dissociation constant slightly increases with decreasing gas temperature, yielding an apparent negative activation energy $\left(1000 \leqslant T_{\mathrm{g}} \leqslant 2000 \mathrm{k}\right)$.

To understand these results, we have reported in figure 5 the $N_{v}$ distributions at different gas temperatures. It should be noted that these distributions are very similar, even though the population densities of higher vibrational levels $(v>30)$, which are responsible of the dissociation rate, decrease with increasing gas temperature. This is due : I) to the fact that the V-T processes, which tend to depopulate the higher vibrational levels, increase their importance with increasing gas temperature; ii) the forward V-V processes, at low gas temperature, present rate coefficients much larger than the reverse ones, due to the fact that the Boltzmann factor which relates for- 


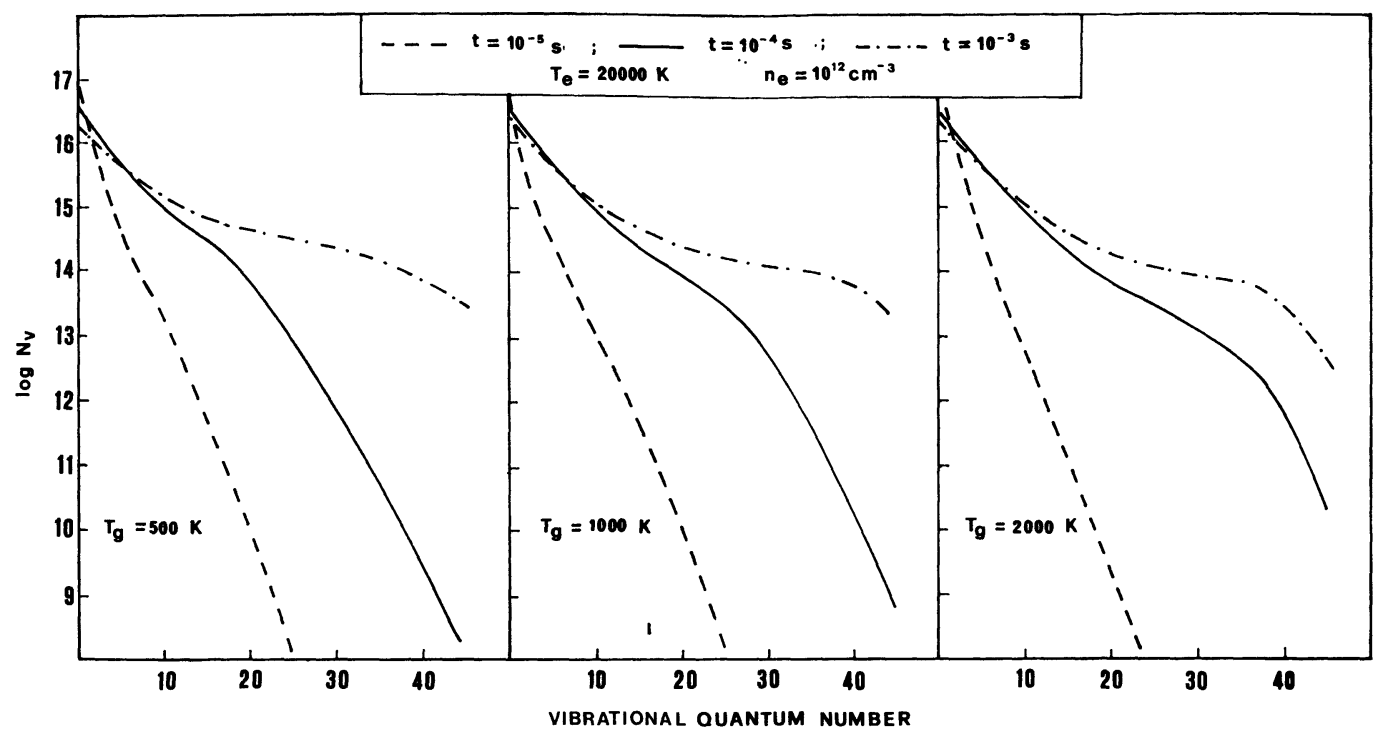

Fig. 5. - Population densities $\left(\log N_{v}\right)$ as a function of vibrational quantum number at different times.

ward and reverse rate coefficients is small at low gas temperature. This last point is made clear by writing the ratio between forward and backward rate coefficients for the near resonant V-V processes :

$$
P_{v, v-1}^{v, v+1} / P_{v-1, v}^{v+1, v}=\exp \left(2 E_{10} \delta / k T_{\mathrm{g}}\right) .
$$

Point i) can be understood by looking at table III, where the V-T and V-V contributions to $v_{\mathrm{d}}$ have been reported at different $T_{\mathrm{g}}$. One can note that the V-T

\section{TABLE III}

Values of the terms $N_{\mathrm{N}_{2}} N_{v} P_{v^{\prime}, v^{\prime}+1}\left(\mathrm{~cm}^{-3} \mathrm{~s}^{-1}\right)$

$$
\text { (a) and }
$$

$$
\sum_{v=1}^{v^{\prime}} N_{v} P_{v, v-1}^{v^{\prime}, v^{\prime}+1} N_{v^{\prime}}\left(\mathrm{cm}^{-3} \mathrm{~s}^{-1}\right)
$$

\section{of equation (2) at different gas temperatures}

$$
\left(N_{\mathrm{N}_{2}}=9.65 \times 10^{16} \mathrm{~cm}^{-3}\right)
$$

$\begin{array}{rcc}T_{\mathrm{g}}(\mathrm{K}) & a & b \\ \overline{5} & - & - \\ 1000 & 1.8(16) & 1.6(19) \\ 2000 & 6.1(17) & 1.8(19) \\ & 6.0(18) & 1.3(19)\end{array}$

$1.8(16)=1.8 \times 10^{16}$

processes are not important at $T_{\mathrm{g}}=500 \mathrm{~K}$, while their importance strongly increases at $2000 \mathrm{~K}$. It should be noted that at $T_{\mathrm{g}}=500 \mathrm{~K}$ the dissociation constant is slightly less than the corresponding values at $T_{\mathrm{g}}=1000,2000 \mathrm{~K}$, since the low temperature increase of $N_{v}$ populations of higher vibrational levels is compensated by the corresponding increase of the rate coefficients appearing in equation (2).
Figure 6 shows the influence of pressure on $k_{\mathrm{d}}^{\mathbf{s}}$ at $n_{\mathrm{e}}=10^{12} \mathrm{~cm}^{-3}$. The behaviour of $k_{\mathrm{d}}^{\mathrm{s}}$ with pressure as given in figure 6 is completely different from that

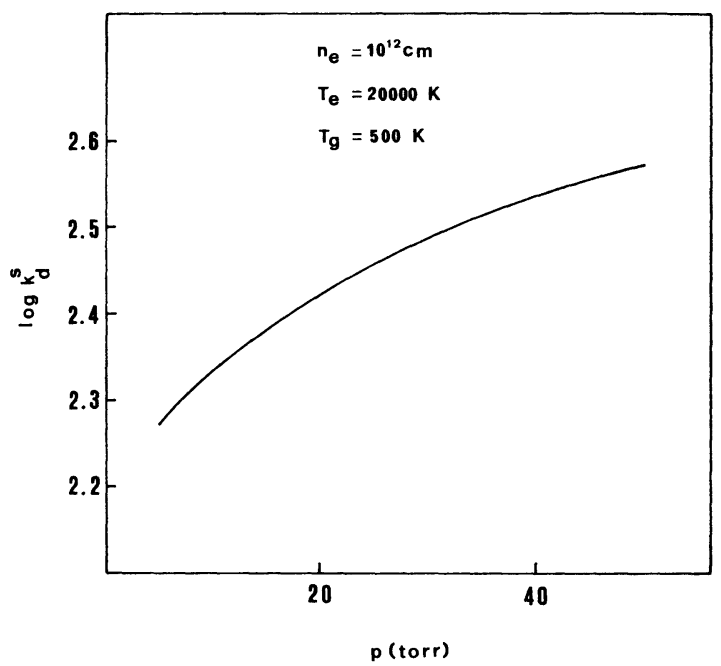

FIG. 6. - Values of $\log k_{\mathrm{d}}^{\mathrm{s}}$ as a function of pressure (torr).

found for $\mathrm{H}_{2}$, where $k_{\mathrm{d}}^{\mathrm{s}}$ strongly decreased with increasing pressure. The present results can be attributed to the small importance of V-T exchanges in affecting the $N_{v}$ distribution at $T_{\mathrm{g}}=500 \mathrm{~K}$. It should be pointed out that at 50 torr and $T_{\mathrm{g}}=500 \mathrm{~K}$ the term $N_{\mathrm{N}_{2}} N_{v^{\prime}} P_{v^{\prime}, v^{\prime}+1}$ is small as compared to

$$
\sum_{v=1}^{v^{\prime}} P_{v, v-1}^{v^{\prime}, v^{\prime}+1} N_{v} N_{v^{\prime}}
$$

This last term increases with pressure, leading to an increase in the dissociation rate. The small increase of $k_{\mathrm{d}}^{\mathrm{s}}$ with pressure (Fig. 6) is due to the fact that at $n_{\mathrm{e}}$ constant the pumping rate for molecule at 5 torr 
is smaller than the corresponding one at 50 torr. A linear increase of $k_{\mathrm{d}}^{\mathrm{s}}$ with pressure is then expected at $p / n_{\mathrm{e}}=$ constant. This is confirmed in table IV $a$ where $k_{\mathrm{d}}^{\mathrm{s}}$ is reported as a function of $n_{\mathrm{e}}$ at $p=50$ torr. It should be noted that $k_{\mathrm{d}}^{\mathrm{s}}$ (50 torr, $n_{\mathrm{e}}=10^{13} \mathrm{~cm}^{-3}$ ) is about 10 times greater than the corresponding value at 5 torr and $n_{\mathrm{e}}=10^{12} \mathrm{~cm}^{-3}$. Table IVb reports $k_{\mathrm{d}}^{\mathrm{s}}$ values as a function of temperature. The increase of $k_{\mathrm{d}}^{\mathrm{s}}$ with $T_{\mathrm{e}}\left(T_{\mathrm{e}}<20000 \mathrm{~K}\right)$ is due to the increase of e-V rate coefficients with $T_{\mathrm{e}}$ (see table I).

\section{TABLE IV $a$}

Values of $k_{\mathrm{d}}^{\mathbf{s}}\left(s^{-1}\right)$ as a function of electron density $n_{\mathrm{e}}\left(\mathrm{cm}^{-3}\right)\left(T_{\mathrm{e}}=20000, T_{\mathrm{g}}=500 \mathrm{~K}\right)$

\begin{tabular}{ccc}
$p$ (torr) & $n_{\mathrm{e}}$ & \multicolumn{1}{c}{$k_{\mathrm{d}}^{\mathrm{s}}$} \\
\hline 50 & $10^{12}$ & 370.0 \\
50 & $10^{13}$ & 1830.0 \\
5 & $10^{12}$ & 186.0
\end{tabular}

\section{TABLE IV $b$}

Values of $k_{\mathrm{d}}^{\mathrm{s}}\left(s^{-1}\right)$ as a function of electron temperature $(K)\left(n_{\mathrm{e}}=10^{12} \mathrm{~cm}^{-3}\right.$, $T_{\mathrm{g}}=500 K, p=5$ torr $)$

$\begin{array}{cr}\frac{T_{\mathrm{e}}}{10000} & \frac{k_{\mathrm{d}}^{\mathrm{s}}}{46.0} \\ 20000 & 186.0\end{array}$

The present results can be compared with similar models proposed in the literature by Caledonia and Center [12] and by Gordiets et al. [13]. According to the first authors, the non-equilibrium vibrational population of diatomic species is made up of three ranges : 1) the first controlled by a Treanor distribution [14] at a given vibrational temperature

$$
\theta_{1}=E_{10} / k \ln \left(N_{0} / N_{1}\right) \text {; }
$$

2) a plateau region in which the $V-V$ near resonart processes dominate the distribution;
3) a deactivation region in which the $N_{v}$ distribution follows a Boltzmann law at the gas temperature.

The onset of this deactivation region occurs at a vibrational quantum number $v *$ given by [12]

$$
\frac{P_{v^{*}, v^{*}-1}}{P_{1,2}^{1,0} v^{*}} \simeq \frac{v_{\mathrm{I}} N_{v_{\mathrm{I}}}}{N_{\mathrm{M}_{2}}} \exp \left(-2 E_{10} \delta / k T_{\mathrm{g}}\right)
$$

where $N_{\mathrm{I}}$ is the population density corresponding to the minimum of the Treanor distribution

$$
v_{\mathrm{I}}=T_{\mathrm{g}} /\left(2 \delta \theta_{1}\right)+0.5 \text {. }
$$

Values of $v^{*}$ and $v_{\mathrm{I}}$, calculated by means of equations (9) and (10) with the aid of $\theta_{1}$ and $N_{v_{I}}$ values taken from the numerical calculation at the maxima of $k_{\mathrm{d}}^{\mathrm{s}}$, have been reported in table $\mathrm{V}$ at different $T_{\mathrm{g}}$.

\section{TABLE V}

Values of the vibrational quantum numbers $v_{\mathrm{I}}$ (see Eq. (10)) $v^{*}$ (see Eq. (9)) and $v^{* *}$ (see Eq. (11e)) at different $T_{\mathrm{g}}\left(n_{\mathrm{e}}=10^{12} \mathrm{~cm}^{-3}, N_{\mathrm{N}_{2}}=9.65 \times 10^{16} \mathrm{~cm}^{-3}\right.$ $\left.T_{\mathrm{e}}=20000 \mathrm{~K}\right)$

$$
\begin{array}{cccccccc}
T_{\mathrm{g}}(\mathrm{K}) & \theta_{1} & (\mathrm{~K}) & v_{\mathrm{I}} & v^{*} & v^{* *} & \delta_{\mathrm{VT}}(a) & \delta_{\mathrm{VV}}(a) \\
- & - & - & - & - & - & - \\
500 & \sim 7630 & \sim 6 & >45 & \sim 69 & 0.311 & 0.296 \\
2000 & \sim 8200 & \sim 20 & >45 & \sim 57 & 0.159 & 0.144
\end{array}
$$

$\left({ }^{a}\right)$ See text.

One can note that $v^{*}$ is always greater than the last bound level of nitrogen molecule $\left(v^{\prime}=45\right)$. As a consequence the Caledonia and Center criterion should not predict Boltzmann tails in the nitrogen system under the conditions studied in the present work, in agreement with our calculations.

The small tails $(v>40)$ present in our calculations do not follow a Boltzmann law at $T_{\mathrm{g}}$ and are probably due to the dissociation reaction which is not considered in the Caledonia and Center model.

The Gordiets et al. approach gives the following formulas for calculating the normalized population density of vibrational levels under non-equilibrium - 1 ditions.

$$
\begin{array}{ll}
f(v)=f(0) \exp \left\{-v\left|E_{1} / \theta_{1}-(v-1) \Delta E / T_{\mathrm{g}}\right|\right\} & v<v_{\mathrm{I}} \\
f(v)=\frac{C}{v+1}-\frac{P_{1,0} \exp \left(\delta_{\mathrm{VT}} v\right)}{P_{1,0}^{0,1} \times \delta_{\mathrm{VT}}(v+1)} & v_{\mathrm{I}} \leqslant v \leqslant v^{* *}
\end{array}
$$

where $E_{1}$ and $\Delta E$ are our $E_{10}$ and $\delta$ values expressed in $\mathrm{K}, \delta_{\mathrm{VT}}$ and $\delta_{\mathrm{VV}}$ which enters in $x$ are parameters appearing in the $\mathrm{V}-\mathrm{T}$ and $\mathrm{V}-\mathrm{V}$ rate constants, while $C$ and $x$ can be calculated from the equations reported in [13]. Values of $\delta_{\mathrm{vT}}$ and $\delta_{\mathrm{vv}}$, which have been reported for some cases in table $\mathrm{V}$, have been obtained by matching our formulas (Eqs. (5)) with the corresponding ones used by Gordiets et al. (see Eqs. (2-3) of ref. [13]). Values of $v^{* *}$ (see [13]) for $T_{\mathrm{g}}=500 \mathrm{~K}$ and $2000 \mathrm{~K}$ have been reported in table $\mathrm{V}$. Once again one can note that these values are greater than the last bound level of the nitrogen molecule, in agree- 
ment with the Caledonia and Center criterion and with our calculations. A good agreement (see table VI) is observed up to vibrational levels far from dissociation $(v \leqslant 30)$, while differences up to a factor of 100 can be noted above this level. It should be noted that Gordiets formulas for $k_{\mathrm{d}}\left(\theta_{1}\right)$ cannot be used in the present calculations, because of $v^{* *}>v^{\prime}$.

\section{TABLE VI}

A comparison of the normalized population densities calculated by the Gordiets et al. method (see Eqs. $(11 a-b))$ and the corresponding values calculated in the present work $\left(N_{\mathrm{N}_{2}}=9.65 \times 10^{16} \mathrm{~cm}^{-3}, T_{\mathrm{e}}=\right.$ $\left.20000 \mathrm{~K}, n_{\mathrm{e}}=10^{12} \mathrm{~cm}^{-3}\right)$

$\begin{array}{rcc}v & f(v)\left(^{a}\right) & f(v)\left(^{b}\right) \\ \overline{0} & - & - \\ 5 & 0.276 & 0.339 \\ 10 & 0.043 & 0.053 \\ 20 & 1.0(-2) & 1.4(-2) \\ 35 & 2.0(-3) & 3.0(-3) \\ 45 & 7.1(-4) & 1.7(-3) \\ & 4.8(-5) & 1.2(-3)\end{array}$

( $\left.{ }^{a}\right)$ Present work.

(b) Equations $(11 a-b)$.

$1.0(-2)=1.0 \times 10^{-2}$.

It is interesting to compare the present $k_{\mathrm{d}}^{\mathrm{s}}$ values with the corresponding values for the direct electronic mechanism from the ground vibrational level

$$
\begin{gathered}
\left(k_{\mathrm{d}}^{\mathrm{e}}(v=0)=k_{\mathrm{d}}^{\mathrm{e}}\right) \\
\mathrm{e}+\mathrm{N}_{2}\left(\mathrm{X}^{1} \Sigma_{\mathrm{g}} v=0\right) \rightarrow \mathrm{e}+\mathrm{N}_{2}^{*} \rightarrow \mathrm{e}+2 \mathrm{~N} .
\end{gathered}
$$

The $k_{\mathrm{d}}^{\mathrm{e}}$ values, obtained by numerical integration of the experimental cross sections of Winters [15] over a Maxwellian distribution function for the electrons, are presented in figure 4 . One can note that the present $k_{\mathrm{d}}^{\mathrm{s}}$ values are always greater than the corresponding $k_{\mathrm{d}}^{\mathrm{e}} n_{\mathrm{e}}\left(\mathrm{s}^{-1}\right)$ values. It should be pointed out that the use of a Maxwellian distribution function for calculating $k_{\mathrm{d}}^{\mathrm{e}}$ is a poor approximation, because the values of the actual distribution function strongly deviate from the Maxwellian in the energy range important for the calculation of $k_{\mathrm{d}}^{\mathrm{e}}$ (i.e. $u>10 \mathrm{eV}$, see Fig. 4 of Ref. [16]).

These deviations should increase $k_{\mathrm{d}}^{\mathrm{e}}$, since the Maxwellian assumption underestimates the tail of the distribution function (see Ref. [16]). Another factor which should increase $k_{\mathrm{d}}^{\mathrm{e}}$ is the insertion of the higher vibrational levels in the direct electronic mechanism. Due to the lack of informations on the relevant cross sections, the following scaling law has been used to test the sensitivity of $\overline{k_{\mathrm{d}}^{\mathrm{e}}}$ on the higher vibrational levels

$$
k_{\mathrm{d}}^{\mathrm{e}}(v)=k_{\mathrm{d}}^{\mathrm{e}}(v=0)(v+1) .
$$

The corresponding $\overline{k_{\mathrm{d}}^{\mathrm{e}}}$ values have been obtained by averaging the different contributions with the known vibrational distribution according to

$$
\overline{k_{\mathrm{d}}^{\mathrm{e}}}=\sum_{v=0}^{v^{\prime}} N_{v} k_{\mathrm{d}}^{\mathrm{e}}(v) / N_{\mathrm{N}_{2}} .
$$

With this assumption $\overline{k_{\mathrm{d}}^{\mathrm{e}}}$ has been found to be 6 times greater than the corresponding $k_{\mathrm{d}}^{\mathrm{e}}(v=0)$ value $\left(n_{\mathrm{e}}=10^{12} \mathrm{~cm}^{-3}, T_{\mathrm{e}}=20000 \mathrm{~K}, T_{\mathrm{g}}=500 \mathrm{~K}\right.$, $p=5$ torr).

4. Accuracy of the results. - The accuracy of the present results will be discussed in connection with the different assumptions made in the calculation.

In particular :

1) The use of SSH theory for calculating V-T and $\mathrm{V}-\mathrm{V}$ rate coefficients, which, despite its wide use, is known to be a poor approximation (see for ex. [17]).

2) The extension of these cross sections, as well as those of $\mathrm{e}-\mathrm{V}$ processes, to the continuum.

3) The use of a Maxwellian distribution function for calculating the rate coefficients of electron vibrational processes.

4) The neglect of the recombination process as well as the influence of atoms on the dissociation constant.

5) The number of vibrational levels considered in the present study.

The computer program has been succesfully tested by comparing our results with the corresponding ones found by Polak [18].

To investigate the sensitivity of the present values to the V-T and V-V rate coefficients, we have varied some of the calculations $\left(T_{\mathrm{e}}=20000 \mathrm{~K}, T_{\mathrm{g}}=500 \mathrm{~K}\right.$, $n_{\mathrm{e}}=10^{12} \mathrm{~cm}^{-3}, p=5$ torr) i) by increasing by a factor 10 all V-T cross sections; ii) by decreasing by the same factor all V-V cross sections. The response of $N_{v}$ and $k_{\mathrm{d}}^{\mathrm{s}}$ to this change can be appreciated from figure 7 and table VII. It should be noted that the results are completely insensitive to the variation in the V-T cross sections, while the corresponding V-V change affects proportionally the results.

This behaviour can be explained by the fact that the $\mathrm{N}_{2}$ system, as already pointed out, practically does not present tails in the $N_{v}$ distribution, due to the small importance of V-T cross sections. A factor 10 of change in these cross sections does not modify the situation, while a similar change in the V-V cross sections directly affects $P_{v, v-1}^{v^{\prime}, v^{\prime}+1}$ appearing in equation (2) without appreciably altering the $N_{v}$ distribution (see Fig. 7). It is evident, however, that at $T_{\mathrm{g}}=2000 \mathrm{~K}$ the $\mathrm{V}-\mathrm{T}$ cross sections are important in affecting the $N_{v}$ distribution. The use of SSH cross sections for calculating the rate coefficients connecting bound states and the continuum (point 2), which is usually done in this kind of calculations, can be 


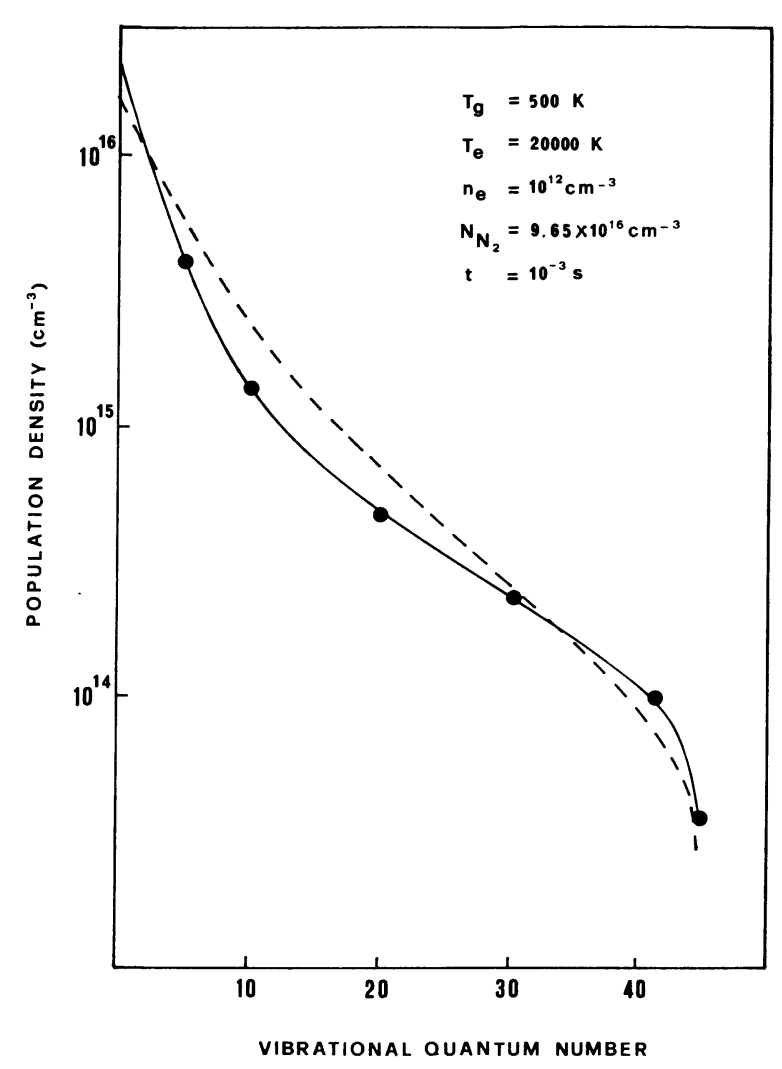

FIG. 7. $-N_{v}$ distributions calculated with different assumptions (- normal V-T and V-V rate coefficients; --- V-V/10; $\mathrm{V}-\mathrm{T} \times 10$ ).

\section{TABLE VII}

Values of $k_{\mathbf{d}}^{\mathbf{s}}\left(s^{-1}\right)$ obtained under different assumptions on $V-V$ and $V$ - $T$ cross sections $\left(T_{\mathrm{g}}=500 \mathrm{~K}\right.$, $T_{\mathrm{e}}=20000 \mathrm{~K}, N_{\mathrm{N}_{2}}=9.65 \times 10^{16} \mathrm{~cm}^{-3}, n_{\mathrm{e}}=10^{12}$ $\mathrm{cm}^{-3}$ )
$\left({ }^{a}\right)$
$\overline{186.0}$
$\left({ }^{b}\right)$
180.0
( $\left.{ }^{a}\right)$ Present results.
(b) V-T rate coefficients increased by a factor 10.
(c) V-V rate coefficients decreased by a factor 10 .

(c)

only justified by the lack of information on these cross sections (especially for V-V transitions).

As for point 3, one can say that in the actual discharge conditions the electron distribution function in $\mathrm{N}_{2}$ strongly deviates from a Maxwellian one especially for energies greater than $4 \mathrm{eV}$ (see Fig. 4 of ref. [16]). At the lower energies which are important in the calculation of $\mathrm{e}-\mathrm{V}$ rate coefficients, the ratio between the actual distribution function and the Maxwellian one presents an oscillatory behaviour. As a consequence one should expect a compensation in the e-V rate coefficients calculated by using Maxwell and non-Maxwell distribution functions due to the integrated nature of these coefficients.
This can be indirectly appreciated by comparing the effective electron vibrational excitation rates calculated by Nighan and the corresponding quantities calculated assuming a Maxwellian distribution function (see table I). It should be noted however that the superelastic collisions can alter the electron distribution function especially at high vibrational temperatures (see Fig. 5 of [16]). An estimation of the relevant energy decay channels (V-T deactivation, superelastic collisions, dissociation) based on equations (12), (22) of ref. [13] shows that the superelastic collisions and the dissociation reaction are the most important energy loss mechanisms for the conditions studied in the present work. This means that a portion of the pumping energy is returned to the electrons via superelastic collisions, thereby indicating that a self consistent computation of dissociation rates can be achieved only by coupling the Boltzmann equation for the electron distribution function to the system of vibrational master equations. This coupling is outside the scope of the present work and we will not pursue it any longer in the present paper.

As for the neglect of the recombination process (point 4) in our master equation, this can be justified by the fact that $k_{\mathrm{d}}(t)$ achieves its stationary value when the concentration of dissociated molecules does not exceed $10 \%$. It should be pointed out, however, that for times greater than $10^{-3} \mathrm{~s}$ the recombination process strongly affects both the dissociation constant of nitrogen and the $k_{\mathrm{d}}(t)$ values.

The number of vibrational levels considered in the present work should not affect the dissociation constant $k_{\mathrm{d}}^{\mathrm{s}}$ especially in the electrical conditions $\left(n_{\mathrm{e}} \neq 0\right)$. In these conditions in fact the lack of a true Boltzmann tail ensures small variations in the population densities of last vibrational levels. The addition of some levels, therefore, does not appreciably modify $k_{\mathrm{d}}^{\mathrm{s}}$.

5. Conclusions. - The results reported show that high dissociation rates can be predicted on the basis of a ladder climbing model of the vibrational levels of ground electronic state of diatomic species.

The accuracy of the present results depends on the set of V-T, V-V and e-V cross sections as well as on the different hypothesis made in the calculation (see points 1-5 of section 4). Moreover the inclusion of rotational processes as well as the multiple quantum transfers in heavy particles collisions should improve the present model.

The fact that the V-T deactivation in the nitrogen system can be neglected means that the dissociation is approximately balanced by the net pumping rate from electrons. From this it follows that our calculated rates can be only as accurate as the pumping rates utilized. These last quantities, however, depend on the superelastic collisions which alter the electron distribution function (and the pumping rates) as already pointed out in section 5 . 
As for the comparison between the present mechanism and the direct one, we can say that the present results indicate our $k_{\mathrm{d}}^{\mathrm{s}}>k_{\mathrm{d}}^{\mathrm{e}} n_{\mathrm{e}}$, even though some assumptions (Maxwellian distribution function, neglection of the direct dissociation mechanism from the higher vibrational levels) could underestimate $k_{\mathrm{d}}^{\mathrm{e}} n_{\mathrm{e}}$, while some cross sections (i.e. the V-V ones) could overestimate our $k_{\mathbf{d}}^{\mathbf{s}}$ values.

Despite these uncertitudes, however, we believe that the present mechanism must be taken into account in the dissociation of nitrogen either for its own importance as discussed in the previous pages, or for preparing the higher vibrational levels of the ground electronic state to jump to dissociative electronic states. The present results, therefore, must be considered as a first step toward the understanding of the nitrogen dissociation in electrical discharges.

Acknowledgements. - The authors thank Prof. D. Trigiante for his contribution to the numerical part of this work and Prof. E. Molinari for helpful discussions and comments.

We want also to thank the referees for their helpful comments, which have improved the original manuscript.

Drawings are by U. Farella.

\section{References}

[1] Capitelli, M., Dilonardo, M. and Molinari, E., Chem. Phys. 20 (1977) 417.

[2] Kewley, D. J., J. Phys. B 8 (1975) 2565.

[3] Bray, K. N. C., MIT Fluid Mechanics Lab. Rept. No. 67-3 (1967).

[4] KeCk, J. and Carrier, G., J. Chem. Phys. 43 (1965) 2284.

[5] Appleton, J. P., J. Chem. Phys. 44 (1967) 3231.

[6] Kiefer, J. H., J. Chem. Phys. 57 (1972) 1938.

[7] Engelhardt, A. G., Phelps, A. V. and Risk, C. G., Phys. Rev. 135 (1964) A 1566.

[8] Trigiante, D., Computing, in press.

[9] Kewley, D. J. and Hornung, H. G., Chem. Phys. Lett. 25 (1974) 531

10] Appleton, J. P., Steinberg, M. and Liquornik, D., $J$. Chem. Phys. 48 (1968) 599.
[11] Valance, W. G., Schlag, E. W. and Elwood, J. P., $J$. Chem. Phys. 47 (1967) 3284.

[12] Caledonia, G. E. and Center, R. E., J. Chem. Phys. 55 (1971) 552.

[13] Gordiets, B. F., Mamedov, S. S. and Shelepin, L., Sov. Phys. JETP 40 (1975) 640.

[14] Treanor, C. E., Rich, J. M. and Rehm, R. G., J. Chem. Phys. 42 (1968) 1798.

[15] Winters, H. F., J. Chem. Phys. 44 (1966) 1472.

[16] Nighan, W. L., Phys. Rev. A5 (1970) 1989.

[17] See for example Miss, F. H., J. Chem. Phys. 40 (1964) 523 ; MCKenzie, R., J. Chem. Phys. 64 (1976) 1498 ; VerTER, M. and Rabitz, H., J. Chem. Phys. 64 (1976) 293.

[18] Polak, L., Pure Appl. Chem. 39 (1974) 307. 